\title{
Normative Data for the Logical Memory Subtest of the Wechsler Memory Scale-IV in Middle-Aged and Elderly Korean People
}

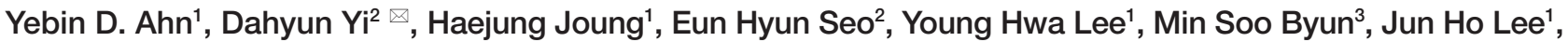 \\ So Yeon Jeon', Jun-Young Lee ${ }^{4,5}$, Bo Kyung Sohn ${ }^{6}$, Dong Young Lee $e^{1,3,5} \bowtie$, for the KBASE Research Group* \\ 1'Department of Neuropsychiatry, Seoul National University Hospital, Seoul, Republic of Korea \\ ${ }^{2}$ Institute of Human Behavioral Medicine, Medical Research Center, Seoul National University, Seoul, Republic of Korea \\ ${ }^{3}$ Premedical Science, College of Medicine, Chosun University, Gwangju, Republic of Korea \\ ${ }^{4}$ Department of Neuropsychiatry, SMG-SNU Boramae Medical Center, Seoul, Republic of Korea \\ ${ }^{5}$ Department of Psychiatry, Seoul National University College of Medicine, Seoul, Republic of Korea \\ ${ }^{6}$ Department of Psychiatry, Sanggye Paik Hospital, Inje University College of Medicine, Seoul, Republic of Korea
}

Psychiatry Investig 2019;16(11):793-799

hhttps://doi.org/10.30773/pi.2019.0061

Unfortunately, two co-authors' and a corresponding author's affiliations were incorrectly listed in the original publication of this article. The correct affiliations of the authors are as follows:

Eun Hyun Seo; ${ }^{3}$ Premedical Science, College of Medicine, Chosun University, Gwangju, Republic of Korea Min Soo Byun; ${ }^{2}$ Institute of Human Behavioral Medicine, Medical Research Center, Seoul National University, Seoul, Republic of Korea Dong Young Lee; ${ }^{1}$ Department of Neuropsychiatry, Seoul National University Hospital, Seoul, Republic of Korea

${ }^{2}$ Institute of Human Behavioral Medicine, Medical Research Center, Seoul National University, Seoul, Republic of Korea

${ }^{5}$ Department of Psychiatry, Seoul National University College of Medicine, Seoul, Republic of Korea

The complete and corrected author list and affiliations are therefore:

Yebin D. Ahn ${ }^{1}$, Dahyun $\mathrm{Yi}^{2}{ }^{凶}$, Haejung Joung1', Eun Hyun $\mathrm{Seo}^{3}$, Young Hwa Lee1, Min Soo Byun², Jun Ho Lee', So Yeon Jeon ${ }^{1}$, Jun-Young Lee ${ }^{4,5}$, Bo Kyung Sohn ${ }^{6}$, Dong Young Lee ${ }^{1,2,5} \bowtie$, for the KBASE Research Group*

1 Department of Neuropsychiatry, Seoul National University Hospital, Seoul, Republic of Korea

${ }^{2}$ Institute of Human Behavioral Medicine, Medical Research Center, Seoul National University, Seoul, Republic of Korea

${ }^{3}$ Premedical Science, College of Medicine, Chosun University, Gwangju, Republic of Korea

${ }^{4}$ Department of Neuropsychiatry, SMG-SNU Boramae Medical Center, Seoul, Republic of Korea

${ }^{5}$ Department of Psychiatry, Seoul National University College of Medicine, Seoul, Republic of Korea

${ }^{6}$ Department of Psychiatry, Sanggye Paik Hospital, Inje University College of Medicine, Seoul, Republic of Korea

(a) This is an Open Access article distributed under the terms of the Creative Commons Attribution Non-Commercial License (http://creativecommons.org/licenses/bync/4.0) which permits unrestricted non-commercial use, distribution, and reproduction in any medium, provided the original work is properly cited. 